\title{
Rapamycin reduces Drosophila longevity under low nutrition
}

\author{
Eugenia Villa-Cuesta ${ }^{1} *$ Frances Fan $^{1}$ and David M. Rand ${ }^{2}$ \\ ${ }^{1}$ Department of Biology, Adelphi University. Garden City, NY 11530, USA. ${ }^{2}$ Department of Ecology and \\ Evolutionary Biology, Brown University, Providence, RI 02912, USA. *Corresponding author
}

\begin{abstract}
Rapamycin treatment is considered a pharmacological intervention with the potential to mimic the longevity benefits of dietary manipulations. However, how rapamycin interacts with nutrition is not fully understood. Here we studied the effect of rapamycin on the longevity of Drosophila under a range of dietary conditions. In diets low in nutrients, rapamycin reduced longevity in a dosage-dependent manner. This dosage effect requires some nutrients as rapamycin has no impact on survival under starvation conditions. Under a balanced diet of yeast and sugar, rapamycin had no repeatable beneficial effect on organismal longevity. These results show that the effect of rapamycin on longevity is sensitive to the nutritional environment and it can reduce lifespan when nutrients are limited.
\end{abstract}

KEYWORDS: Nutrition, Longevity, Rapamycin

\section{INTRODUCTION}

Studies in mammals and invertebrates have identified rapamycin as a drug that may mimic the benefits of diet restriction and have anti-aging effects (1-8). Rapamycin inhibits the TORC1 and TORC2 complexes of the mammalian target of rapamycin (mTOR) pathway that regulates nutrient sensing, placing rapamycin at the interface between nutrition and longevity control (1-7). The promise of rapamycin as a pharmacological agent that can extend longevity calls for a thorough understanding of the molecular targets and environmental mediators of rapamycin's effect on longevity. In genetically heterogeneous mice, rapamycin treatment can extend the life span of both males and females $(9,10)$. In flies, two independent studies reported longevity effects of rapamycin $(11,12)$. The most comprehensive of those studies showed increased lifespan of flies maintained on food supplemented with concentrations of rapamycin ranging from $50 \mu \mathrm{M}$ to $400 \mu \mathrm{M}$. At a fixed concentration of $200 \mu \mathrm{M}$, a concentration at which flies are expected to incorporate a similar amount of rapamycin as that reported in studies of mice, rapamycin extended longevity in several diets (12). Independently, the second study reported that flies maintained on a nutrient rich food supplemented with 1,10 or $100 \mu \mathrm{M}$ of rapamycin showed no significant differences in life span, but that rapamycin could have detrimental effects at $500 \mu \mathrm{M}$ or higher concentrations (11). Here we undertook an independent study of the effect of rapamycin on Drosophila longevity under a range of dietary conditions.

Since amino acid imbalance is a key determinant of Drosophila lifespan (13), the restriction of food in Drosophila is frequently performed via the dilution of yeast as a protein source. Here we measured the effect of rapamycin on longevity of Drosophila maintained on four different diets with a range of yeast levels $(1,2,3,4$, 8 and $12 \%$ weight/volume) spanning the conditions that extend longevity in previous experiments (14). We further examined the effects of rapamycin on survival on a starvation diet $(0.8 \% \mathrm{w} / \mathrm{v}$ agar in water with no additional nutrients). Rapamycin was added to the diets at different concentrations to explore both dosedependent and presence-absence effects.

\section{MATERIALS AND METHODS}

All stocks were maintained and conducted under standard conditions $\left(25^{\circ} \mathrm{C}, 12 \mathrm{~h}\right.$ light: $12 \mathrm{~h}$ dark $)$ on normal media (2\% yeast, $11 \%$ sugar, $5.2 \%$ cornmeal, agar $0.8 \% \mathrm{w} / \mathrm{v}$ in water and $0.2 \%$ tegosept -methyl 4 hydroxybenzoate, from Sigma- St. Louis, MO, USA). All stocks were density controlled in replicate bottles using 48-hour egg lays by 20 pairs of parents for two generations prior to collection of flies for experimental assays. Adults were mated for 24 hours, anesthetized with moist $\mathrm{CO}_{2}$, sorted by sex and allowed to recover for 24 hours before initiating longevity cages. 100 females were transferred to 1 liter demography cages. Dead flies were recorded and removed every 2 or 3 days, at which time fresh food was provided in a vial with $5 \mathrm{ml}$ of medium. We used three replicate cages for each treatment/genotype. For starvation studies flies were maintained in a $0.8 \%$ Difco bacto-agar in water. $0.1 \mathrm{X} \mathrm{SY}$ food was prepared as described in (12). Rapamycin was purchased from LC Laboratories (Woburn, MA, USA.). Rapamycin was dissolved in ethanol and added to food, as described in (12). 


\section{RESULTS}

\subsection{Longevity effects of rapamycin under different diets}

As expected, dietary restriction of yeast at 2,3 , or $4 \%$ diet causes a repeatable extension of longevity relative to $1 \%$ yeast, in two independent experimental blocks separated by 6 months (Figure 1, A and B). However, the addition of $200 \mu \mathrm{M}$ rapamycin to these diets does not show a consistent effect on longevity. On a diet of $2 \%$ yeast rapamycin extended longevity in one experiment (log rank test: $\left.\chi^{2}=43.9 ; P<0.0001\right)$ but had no effect when repeated, resulting in a highly significant block effect in a proportional hazards model testing for main effects of rapamycin and block $\left(\chi^{2}=43.3 ; P<0.0001\right.$; see supplementary Table 1$)$. On diets of $3 \%$ and $4 \%$ yeast the effects of rapamycin were reversed across repeat experiments, with one block showing non significant effects of rapamycin on these two diets and the second block showing significant effects in the opposite directions (see Figure $1 \mathrm{C}$ and $\mathrm{D}$; $\log$ rank test on $3 \%$ yeast: $\chi^{2}=5.91, P<0.015$; on $4 \%$ yeast $\chi^{2}=11.15$, $P<0.0008)$. In a proportional hazards model, these data showed no main effect of rapamycin or block, but a highly significant rapamycin X block interaction (supplementary Table 1).

Interestingly, in a diet low in nutrients (1\% yeast), rapamycin consistently decreased the mean lifespan in both experimental blocks by $28 \%$ and $16.5 \%$ respectively (see Figure $1 \mathrm{~A}-\mathrm{D}$; $\log$ rank test for $1 \%$ yeast: $\chi^{2}=$ 54.09, $P<0.0001 ; \chi^{2}=13.49, P<0.0002$ for block 1 and 2 (Figure 1C and D), respectively). A proportional hazards model showed a highly significant effect of rapamycin, no effect of block and a weak rapamycin $\mathrm{X}$ block interaction $(P<0.0151)$ that does not withstand correction for multiple tests for these four diets (Figure 1, and supplemental Table 1). Supplementary Table 1 presents a summary of the rapamycin and repeatexperiment (block) effects for each diet condition $(1,2,3$, an $4 \%$ yeast), as revealed by analysis of variance. The $1 \%$ yeast diet is unique in that it is the one condition that shows a highly significant main effect of rapamycin that reduces longevity coupled with no significant main effect of block (repeat experiments are consistent). The other diet conditions, ranging from 2 to $4 \%$ yeast, either show no significant main effect of rapamycin, or significant main effects of block, implying that the rapamycin effects are not repeatable.

In an effort to determine if the longevity-extending effect of rapamycin were restricted to high-nutrient diets, we conducted a third independent longevity assay using $2 \%$ and $8 \%$ yeast. The $2 \%$ treatment was used to provide a reference to the other experimental blocks, since block effects are common in longevity assays. Rapamycin significantly extended the longevity of flies fed both $2 \%$ and $8 \%$ yeast in this third experiment, (log rank test for $2 \%$ yeast: $\left.\chi^{2}=40.663, p<0.001\right]$, for $8 \%$ yeast: $\left.\left[\chi^{2}=24.475, p<0.001\right]\right)$. Analysis of variance for the effects of rapamycin and yeast showed a significant effect of rapamycin treatment in both diets and a modest effect of the yeast diet (ANOVA: treatment [DF: 1, F: 42.332, p<0.001], yeast [DF: 1, F: 4.116, $p=0.043$ ] and yeast*treatment interaction [DF: $1, \mathrm{~F}: 0.165, \mathrm{p}=0.685]$ ) (Figure 1E-F). It should be noted that the mean longevities in this third experiment were higher than in other experimental blocks, but the expected effect of somewhat reduced longevity under richer diet is observed ( $8 \%$ shows shorter longevity than $2 \%$ yeast; see Figure 1E-F). The longevity of the OreR strain of D. melanogaster is known to be rather unresponsive to diet in the 5-15\% range on SY media, relative to other genotypes (15).

We next conducted a fourth independent study using increasing concentrations of rapamycin $(0,5,50$, 100,200 and $400 \mu \mathrm{M}$ ) in $2 \%$ and $12 \%$ yeast diet (Figure 2). Concentrations of rapamycin ranging from 50 to $200 \mu \mathrm{M}$ extended the mean longevity of flies fed $2 \%$ yeast diet, reaching maximum significant extension at 200 $\mu \mathrm{M}$ rapamycin (log rank test for $2 \%$ yeast at $200 \mu \mathrm{M}\left[\chi^{2}=6.435, p<0.01\right]$ ) and decreasing at $400 \mu \mathrm{M}$ (Figure $4 \mathrm{~A}$ and $\mathrm{C}$ ). However, increasing concentrations of rapamycin were not able to extend longevity of flies fed a $12 \%$ yeast diet (Figure 2B and C) suggesting that higher concentrations of rapamycin are needed to extend the longevity of flies fed a very high yeast diet. Although there was not a significant difference in the longevity of the flies fed the yeast diets at $0 \mu \mathrm{M}$ rapamycin, analysis of variance for the effects of rapamycin and yeast showed a significant effect of the yeast diet (ANOVA: diet [DF: 1, F: 8.911, p<0.003]) and yeast*treatment interaction (ANOVA [DF: 1, F: 3.31, $\mathrm{p}=0.006]$ ).

One other thing to note is the more linear shape of the survivorship curves in Figure 2 compared to Figure 1. Since the longevity measurement represented in figure 2 was performed at a different laboratory, we attribute this difference to a genotype $\mathrm{X}$ environment interaction (GxE) (16).

\subsection{Longevity effects of rapamycin under low nutrition diets.}

We hypothesized that low-nutrient diets might require lower concentrations of rapamycin to obtain the beneficial effects on longevity. To test this hypothesis we measured lifespan on $1 \%$ yeast using two standard laboratory strains ( $w^{\text {Dha }}$ and OreR) and rapamycin concentrations ranging from 5 to $200 \mu \mathrm{M}$. At $5 \mu \mathrm{M}$, rapamycin decreased median lifespan of $w^{D h a}$ strain but did not significantly affect the lifespan of OreR, relative to the $0 \mu \mathrm{M}$ control. At higher concentrations, rapamycin decreased the life span of both strains in a dose-dependent manner (Figure 3C). Previous studies reported an extension of longevity by rapamycin in all diets tested, even in those with low nutritional value (12). Because the low nutritional value diet used in that study differs from the diet 
used in our experiments, we repeated the survival analysis using the same low nutritional diet as published by Bjedov et al. 2010 (0.1X SY,(12)). Rapamycin significantly shortened the mean lifespan of both strains when maintained in a $0.1 \mathrm{X}$ SY diet (Figure 3D,E).

\subsection{The effect of rapamycin on starvation resistance}

We also studied the effect of rapamycin under starvation conditions. $w^{\text {Dha }}$ and OreR strains maintained in a starvation diet were exposed to 5,50 and $200 \mu \mathrm{M}$ of rapamycin. Rapamycin did not have any effect on the resistance to starvation of $w^{D h a}$ and OreR flies (Figure 4A, B). Interestingly, $w^{\text {Dha }}$ strain showed an overall higher resistance to starvation than OreR strain (Figure 4C). Several technical points are worth noting for these starvation assays. Bjedov et al (2010) used 1.5\% agar in starvation assays - twice the concentration of our assay - and flies survived 1-2 days longer in their study than what we report here, suggesting that agar may have some nutritional value for Drosophila. Rapamycin has low solubility in water (17), and the amount of rapamycin delivered to flies in the no-nutrient agar-water medium may be lower than in media containing nutrients that could act as co-solvents for rapamycin delivery. However, all rapamycin exposures used ethanol as a co-solvent to enhance solubility(17).

\section{CONCLUSION}

In summary, there are three conclusions from these experiments: 1) Under limited nutrition, rapamycin decreases the longevity of two Drosophila strains in a dosage-dependent manner (Figure 3 ) in two independent low-nutrition diets (1\% yeast and 0.1X SY food). 2) Rapamycin does not consistently extend longevity of Drosophila melanogaster under unrestricted diets (Figure 1 and 2). 3) The detrimental effects of rapamycin are not observed in two Drosophila strains when under starvation conditions (Figure 4). The lack of a detrimental effect of rapamycin under starvation suggests that the pharmacological effects of rapamycin depend upon the nutritional state of the fly. Thus, as a dietary restriction mimetic, the addition of rapamycin to a diet that is already low in nutrients may exacerbate malnutrition and reduce longevity. It may be that decreased longevity is not observed under starvation because rapamycin cannot further restrict dietary nutrients. However, rapamycin may need more time than only a few days to modulate longevity and its effect under starvation might be hindered by the short longevity of starved flies.

Although we did find that rapamycin can extend longevity at balanced diets with non-limiting nutritional conditions (Figure 1), these effects were not repeatable in different experimental blocks. Of the seven longevity experiments conducted on yeast diets in the $2-4 \%$ range, rapamycin extended longevity in three experiments, had no effect in three experiments, and reduced longevity in one experiment (Figure 1). These data suggest that longevity extension by rapamycin under particular diets is not sufficiently repeatable that it can over-ride variable environmental conditions that are typically associated with replication of longevity experiments at different times, even using identical nutrient conditions.

Environmental interactions in rapamycin treatment might also explain the different results described in the literature $(11,12)$. Rapamycin strongly reduces female fecundity (12), and reproductive output typically shows a negative correlation (trade-off) with longevity. The variable evidence for longevity extension by rapamycin in Drosophila may be partly attributable to indirect effects on longevity due to variable inhibition of reproductive effort when nutrients are sufficient to support egg production. The detrimental effects of rapamycin under low nutrients that we describe here may be heightened if the nutrient level is sufficiently low that egg production is already turned off, preventing any further beneficial effect of rapamycin from its inhibitory impact on reproduction. While this trade-off hypothesis is plausible, it remains possible that the distinct results from different experiments represent subtle variation in nutrient levels that confound the delivery or metabolism of rapamycin upstream or downstream of its site of action.

While rapamycin may indeed be a promising drug for the treatment of age related diseases or the extension of lifespan, the complex interactions reported here suggest that the precise nutritional and environmental conditions under which rapamycin may realize this promise are not fully understood. There is ongoing controversy in the field about the generality of the effects of rapamycin $(18,19)$, and it is incumbent on researchers in this field to resolve this uncertainty through independent, replicated experiments. Given the variable results from model organism studies conducted in different laboratories with standard diets, temperatures and growth conditions, the use of rapamycin to treat human ailments will be challenging given the widely varying dietary and environmental conditions to which the general public are exposed. With additional studies of how diet may modify the effects of rapamycin, these challenges may well be overcome. 
Rapamycin reduces Drosophila...

\section{ACKNOWLEDGEMENTS}

We would like to acknowledge Leann Biancani, Marissa Holmbeck and James Mossman for general assistance with these experiments, and Emma Blake, Denise Croote, Julia Donner, Michelle Koh, Russyan Mark Mabeza, Neha Meta, and Matthew Min for work on longevity experiments. This work is supported by Adelphi Faculty Development Grant (to EVC) and National Institutes of Health grant 5R01AG027849-03 (to D.M.R.)

\section{References}

[1]. Lamming DW, Ye L, Sabatini DM, Baur JA. Rapalogs and mTOR inhibitors as anti-aging therapeutics. J Clin Invest; 2013; 123:980-9.

[2]. Kaeberlein M. Longevity and aging. F1000Prime Rep . 2013;5:5.

[3]. Bjedov I, Partridge L. A longer and healthier life with TOR down-regulation: genetics and drugs. Biochem Soc Trans. 2011;39:460-5.

[4]. Kaeberlein M. Resveratrol and rapamycin: are they anti-aging drugs? Bioessays. 2010;32:96-9.

[5]. Sierra F. Rapamycin joins the aging fray: maybe Ponce de Leon visited Rapa Nui, not Florida. J Gerontol A Biol Sci Med Sci. 2010;65:577-9.

[6]. Ingram DK, Zhu M, Mamczarz J, Zou S, Lane M a, Roth GS, et al. Calorie restriction mimetics: an emerging research field. Aging Cell. 2006;5:97-108.

[7]. Le Couteur DG, McLachlan AJ, Quinn RJ, Simpson SJ, de Cabo R. Aging biology and novel targets for drug discovery. J Gerontol A Biol Sci Med Sci. 2012;67:168-74.

[8]. Zhang Y, Bokov A, Gelfond J, Soto V, Ikeno Y, Hubbard G, et al. Rapamycin Extends Life and Health in C57BL/6 Mice. J Gerontol A Biol Sci Med Sci. 2013

[9]. Harrison DE, Strong R, Sharp ZD, Nelson JF, Astle CM, Flurkey K, et al. Rapamycin fed late in life extends lifespan in genetically heterogeneous mice. Nature. 2009;460:392-5.

[10]. Miller RA, Harrison DE, Astle CM, Baur JA, Boyd AR, de Cabo R, et al. Rapamycin, but not resveratrol or simvastatin, extends life span of genetically heterogeneous mice. J Gerontol A Biol Sci Med Sci. 2011;66:191-201.

[11]. Harrison B, Tran TT, Taylor D, Lee S-D, Min K-J. Effect of rapamycin on lifespan in Drosophila. Geriatr Gerontol Int. 2010;10:110-2.

[12]. Bjedov I, Toivonen JM, Kerr F, Slack C, Jacobson J, Foley A, et al. Mechanisms of life span extension by rapamycin in the fruit fly Drosophila melanogaster. Cell Metab.2010;11:35-46.

[13]. Grandison RC, Piper MDW, Partridge L. Amino-acid imbalance explains extension of lifespan by dietary restriction in Drosophila. Nature. 2009;462:1061-4.

[14]. Min K-J, Tatar M. Drosophila diet restriction in practice: do flies consume fewer nutrients? Mech Ageing Dev. 2006;127:93-6.

[15]. Grandison RC, Wong R, Bass TM, Partridge L, Piper MDW. Effect of a standardised dietary restriction protocol on multiple laboratory strains of Drosophila melanogaster. PLoS One. 2009 J4:e4067.

[16]. Zhu C-T, Ingelmo P, Rand DM. $G \times G \times E$ for Lifespan in Drosophila: Mitochondrial, Nuclear, and Dietary Interactions that Modify Longevity. Larsson N-G, editor. PLoS Genet .2014;10:e1004354.

[17]. Simamora P, Alvarez JM, Yalkowsky SH. Solubilization of rapamycin. Int J Pharm. 2001;213:25-9.

[18]. Johnson SC, Martin GM, Rabinovitch PS, Kaeberlein M. Preserving youth: does rapamycin deliver? Sci Transl Med. 2013;5:211fs40.

[19]. Neff F, Flores-Dominguez D, Ryan DP, Horsch M, Schröder S, Adler T, et al. Rapamycin extends murine lifespan but has limited effects on aging. J Clin Invest; 2013;123:3272-91. 


\section{Figures}

A
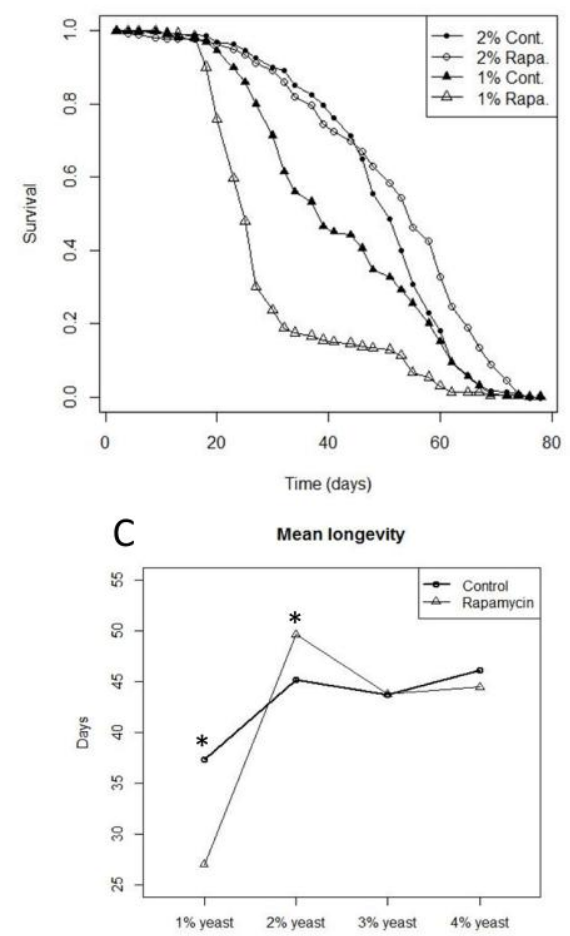

B

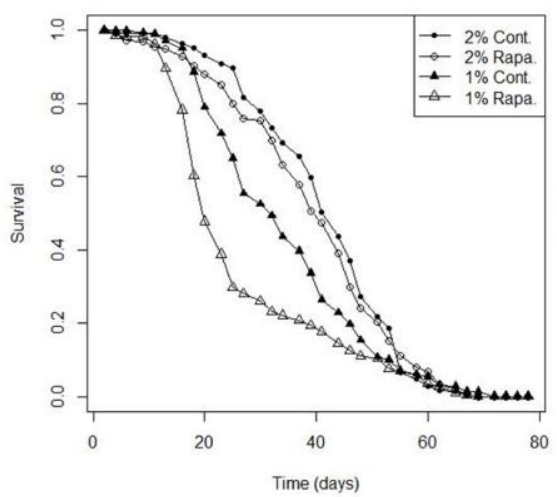

D

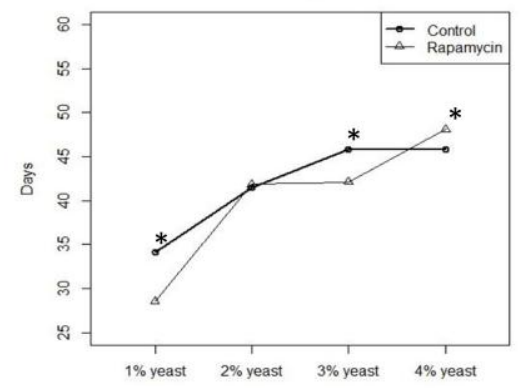

$\mathrm{E}$

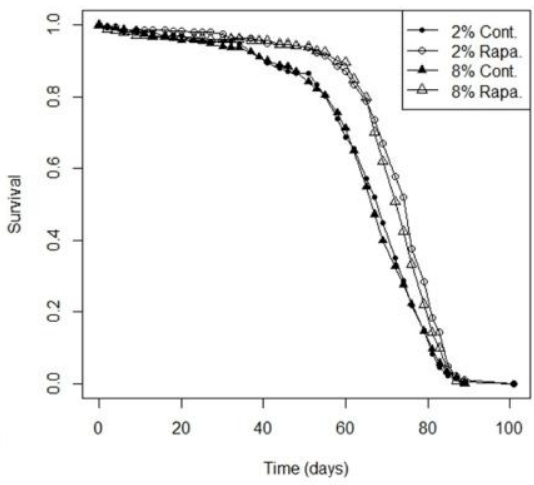

F

Mean longevity

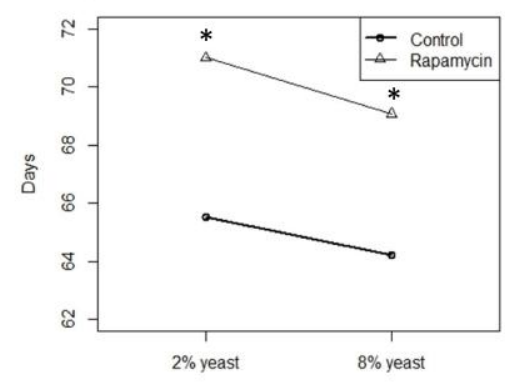

Figure 1. Longevity effects of rapamycin under different diets. Longevity analysis of $D$. melanogaster strain OreR under 2\% (circle) and 1\% yeast food (triangle) exposed to rapamycin (open symbols) and vehicle control (filled symbols). Panels A and B show results from two independent experiments, block 1 and 2, respectively. (C and D) Mean longevity of flies under rapamycin treatment (triangle) and control (circle) in 1, 2, 3 and 4\% yeast food. Experimental block 1 (C) and block 2 (D) (E) Survival analysis of $D$. melanogaster OreR strain under $8 \%$ yeast food with $200 \mu \mathrm{M}$ rapamycin added. (F) Mean longevity values for the data in (E) . $* P<0.05 \log$ rank test.

\begin{tabular}{|c|c|c|c|c|c|c|c|}
\hline \multirow[b]{2}{*}{ Panel } & & \multicolumn{3}{|l|}{ Mean } & \multicolumn{3}{|c|}{ \% life span extension } \\
\hline & & A & B & $\mathbf{E}$ & $\mathbf{A}$ & B & $\mathbf{E}$ \\
\hline \multirow[t]{2}{*}{$1 \%$ yeast } & Control & 37.3 & 34.1 & & & & \\
\hline & Rapa & 27.9 & 28.5 & & $-28 \%$ & $16.4 \%$ & \\
\hline \multirow[t]{2}{*}{$2 \%$ yeast } & Control & 43.3 & 41.4 & 65.5 & & & \\
\hline & Rapa & 45.6 & 41.8 & 71 & $5.1 \%$ & & $8.4 \%$ \\
\hline \multirow[t]{2}{*}{$3 \%$ yeast } & Control & 44.7 & 45.7 & & & & \\
\hline & Rapa & 42.9 & 42.1 & & & $4 \%$ & \\
\hline \multirow[t]{2}{*}{$4 \%$ yeast } & Control & 45.9 & 45.8 & & & & \\
\hline & Rapa & 46.1111 & 47.9 & & & $4.5 \%$ & \\
\hline \multirow[t]{2}{*}{$8 \%$ yeast } & Control & & & 64.2 & & & \\
\hline & Rapa & & & 69 & & & $7.4 \%$ \\
\hline
\end{tabular}


A

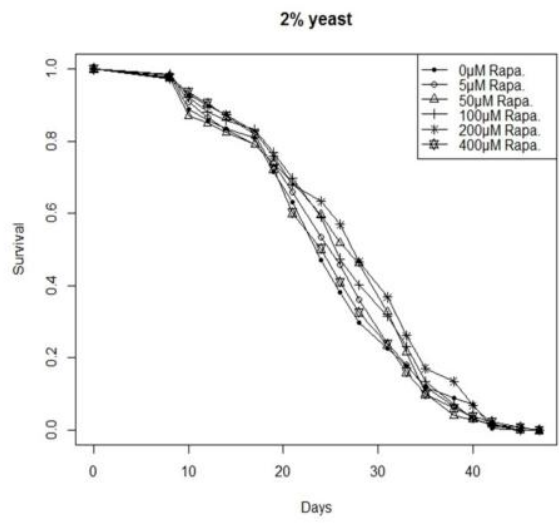

B

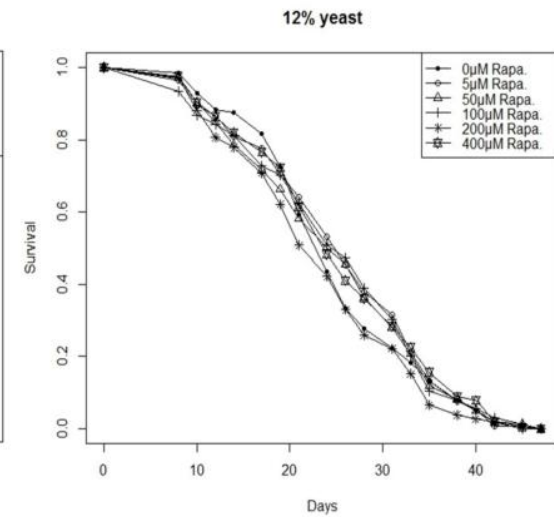

C

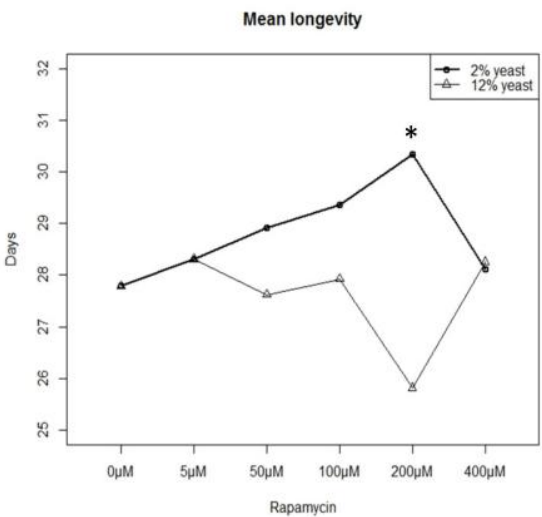

Figure 2.The longevity effect of different rapamycin concentrations.

Longevity analysis of D. melanogaster strain $w^{\text {Dha }}$ under $2 \%(\mathrm{~A})$ and $12 \%$ (B) yeast food exposed to $0,5,50$, 100,200 and $400 \mu \mathrm{M}$. (C) Mean longevity for the data in A and B. $* P<0.05 \log$ rank test for the comparison between 0 and $200 \mu \mathrm{M}$.

\begin{tabular}{|l|l|l|l|l|}
\cline { 2 - 5 } \multicolumn{1}{c|}{} & \multicolumn{2}{|l|}{ Mean } & \multicolumn{2}{c|}{ \% life span extension } \\
\cline { 2 - 5 } \multicolumn{1}{c|}{} & $2 \%$ yeast & $12 \%$ yeast & $\begin{array}{l}2 \% \\
\text { yeast }\end{array}$ & 12\% yeast \\
\hline O uM & 27.8 & 27.8 & & \\
\hline 5 uM & 28.3 & 28.3 & & \\
\hline 50 uM & 28.9 & 27.6 & 4 & \\
\hline 100 uM & 29.4 & 27.9 & 5.7 & \\
\hline 200 uM & 30.3 & 25.8 & 9 & -7.2 \\
\hline 400 uM & 28.2 & 28.2 & & \\
\hline
\end{tabular}



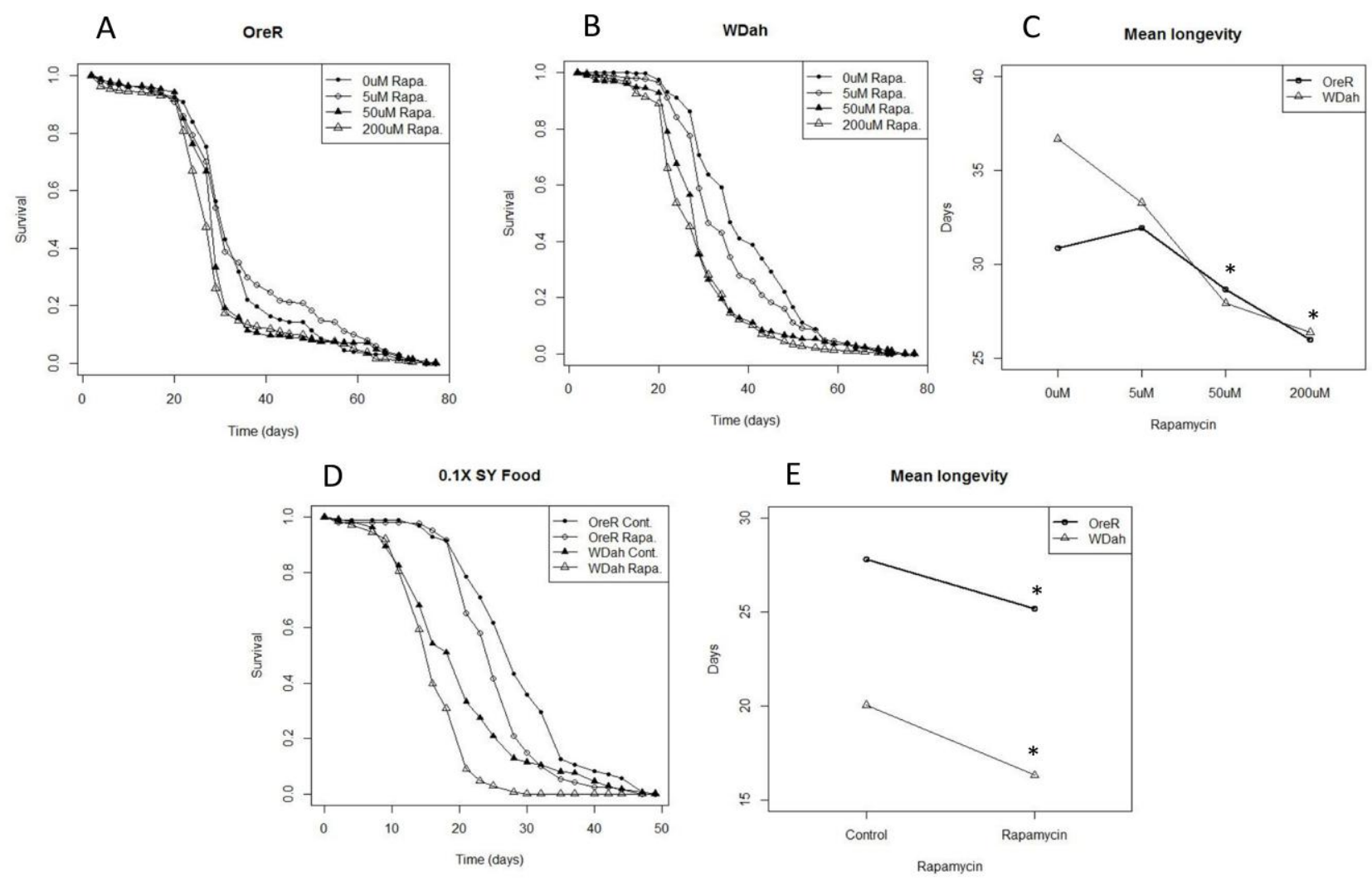

Figure 3. Longevity effects of rapamycin under low nutrition diets. Survival analysis of $D$. melanogaster strains $\operatorname{OreR}(\mathrm{A})$ and $w^{\text {Dha }}$ (B) on $1 \%$ yeast food with $0,5,50$ and $200 \mu \mathrm{M}$ rapamycin added. (C) Mean longevity for the data in A and B. (D) Survival analysis of OreR and $w^{\text {Dha }}$ strains in 0.1X SY food with $200 \mu \mathrm{M}$ rapamycin added. (E) Mean longevity values for the data in (D). $* P<0.05$ log rank test.

\begin{tabular}{|c|c|c|c|c|c|}
\hline & \multicolumn{2}{|l|}{ Mean } & \multicolumn{2}{|c|}{$\begin{array}{ll}\% \text { life } & \text { span } \\
\text { extension }\end{array}$} \\
\hline & & OreR & WDah & OreR & WDah \\
\hline \multicolumn{2}{|l|}{$0 \mathrm{uM}$} & 30.8 & 36.7 & & \\
\hline \multicolumn{2}{|l|}{ 5uM } & 31.9 & 33.2 & 3.5 & -9.5 \\
\hline \multicolumn{2}{|l|}{ 50uM } & 28.65 & 27.9 & -4.5 & -23.9 \\
\hline \multicolumn{2}{|c|}{$200 \mathrm{uM}$} & 26 & 26.36 & -15.5 & -28.2 \\
\hline \multirow{2}{*}{$\begin{array}{l}\text { 0.1SY } \\
\text { OreR }\end{array}$} & Control & 27.8175 & 20.0316 & & \\
\hline & Rapa & 25.1711 & 16.3152 & -9.3 & -18.5 \\
\hline
\end{tabular}



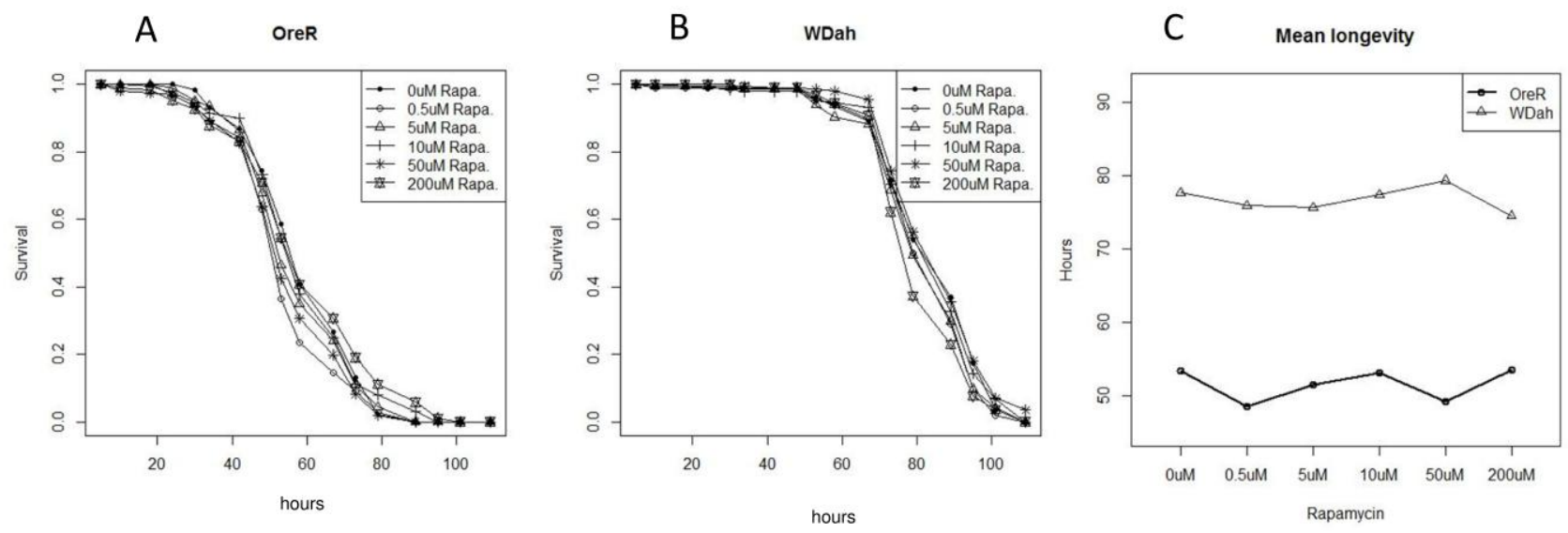

Figure 4.The effect of rapamycin on starvation resistance. Survival analysis of $D$. melanogaster $\operatorname{OreR}(\mathrm{A})$ and $w^{\text {Dha }}(\mathrm{B})$ strains under starvation $(0.8 \%$ agar in water) with $0.55,10,50$ and $200 \mu \mathrm{M}$ rapamycin added. (C) Mean longevity values for the data in (A) and (B). 


\section{Supplementary Table}

Table 1. Analysis of variance for effects of rapamycin and experimental block on Drosophila longevity

\begin{tabular}{|c|c|c|c|c|c|c|c|}
\hline $1 \%$ yeast & DF & L-R $\chi^{2}$ & $P<$ & Term & Risk Ratio & Lower CL & Upper CL \\
\hline Rapamycin & 1 & 49.042 & 0.0000 & Rapamycin 0uM & 0.804 & 0.756 & 0.854 \\
\hline Block & 1 & 0.057 & 0.8107 & Block 2 & 1.007 & 0.948 & 1.071 \\
\hline Rapamycin*Block & 1 & 5.907 & 0.0151 & Rapamycin*Block & 1.078 & 1.015 & 1.146 \\
\hline $2 \%$ yeast & DF & L-R $\chi^{2}$ & $P<$ & Term & Risk Ratio & Lower CL & Upper CL \\
\hline Rapamycin & 1 & 28.938 & 0.0000 & Rapamycin OuM & 1.184 & 1.113 & 1.258 \\
\hline Block & 1 & 43.275 & 0.0000 & Block 2 & 1.227 & 1.155 & 1.304 \\
\hline Rapamycin*Block & 1 & 8.339 & 0.0039 & Rapamycin*Block & 0.916 & 0.862 & 0.972 \\
\hline $3 \%$ yeast & $\mathrm{DF}$ & L-R $\chi^{2}$ & $P<$ & Term & Risk Ratio & Lower CL & Upper CL \\
\hline Rapamycin & 1 & 0.001 & 0.9696 & Rapamycin 0uM & 1.001 & 0.943 & 1.062 \\
\hline Block & 1 & 0.728 & 0.3936 & Block 2 & 0.975 & 0.918 & 1.034 \\
\hline Rapamycin*Block & 1 & 10.882 & 0.0010 & Rapamycin*Block & 0.905 & 0.853 & 0.960 \\
\hline $4 \%$ yeast & $\mathrm{DF}$ & L-R $\chi^{2}$ & $P<$ & Term & Risk Ratio & Lower CL & Upper CL \\
\hline Rapamycin & 1 & 1.037 & 0.3084 & Rapamycin OuM & 1.031 & 0.972 & 1.093 \\
\hline Block & 1 & 24.081 & 0.0000 & Block 2 & 0.861 & 0.812 & 0.914 \\
\hline Rapamycin*Block & 1 & 12.613 & 0.0004 & Rapamycin*Block & 1.112 & 1.049 & 1.179 \\
\hline
\end{tabular}

Proportional hazards analyses were conduced in the JMP statistical package. Two-way models were analyzed separately by diet $(1,2,3$, or $4 \%$ yeast), and results are shown in the left four columns. Risk ratio values are shown in the right four columns, with the reference conditions being 0uM Rapamycin and Block 2 for the two main effects. A lower risk ratio value means that treatment has a lower risk of death, or an extended longevity. These analyses seek to determine the direction and repeatability of the rapamycin effect on longevity.For yeast level of $1 \%$, there is a highly significant rapamycin effect showing a reduced risk ratio (0.804) for the no-rapamycin control. The Block effect is not significant meaning that the two independent experiments gave similar results (the confidence limits (CL) of the risk ratio for Block in the $1 \%$ diet include 1.0 , or no change in risk). The interaction term has a $P$ value $<0.05$, but with 4 tests this is not significant as the critical value should be $0.05 / 4=0.0125$.

In the other diet levels (2, 3, and 4\%), there is either a significant block effect or no significant rapamycin effect, indicating that the effects of rapamycin are not repeatable, or not measurable. The significant interaction terms for 2,3 , and $4 \%$ diets indicate that the difference in longevity between rapamycin and control treatments is significantly different in the two experimental blocks. 